\title{
Interferon regulatory factor 5 and autoimmune lupus
}

\section{Wang-Dong $X u^{1}$, Hai-Feng Pan ${ }^{1}$, Yuekang $X u^{1,2, \dagger}$ and Dong-Qing $Y^{1, *, \dagger}$}

Systemic lupus erythematosus (SLE) is a severe multi-system autoimmune disease, whereas interferon regulatory factor (IRF) 5 belongs to the family of transcription factors that modulate immune system activities. Recently, many lines of investigations suggested that IRF5 gene polymorphisms are closely associated with the disease onset of SLE. Indeed, expressed in B cells, dendritic cells (DCs), monocytes and macrophages, IRF5 could significantly affect these immune cells participating in the pathogenesis of SLE, and numerous studies implied that this transcription factor is mechanistically linked to the disease progression. Here, we comprehensively review the updated evidence indicating the roles of IRF5 in autoimmune lupus. Hopefully, the information obtained will lead to a better understanding of the pathogenesis and development of novel therapeutic strategies for the systemic autoimmune disease.

Systemic lupus erythematosus (SLE) is a systemic autoimmune disease, characterised by autoantibody production, complement activation and immune-complex deposition which eventually lead to tissue and organ damage (Ref. 1). It has now been widely accepted that a combination of genetic and environmental factors might associate with the pathogenesis of SLE. Interferon (IFN) regulatory factors (IRFs) are the best characterised transcriptional regulators of type I IFNs and IFNinducible genes, which are now recognised to play pivotal roles in the regulation of many facets of innate and adaptive immune responses (Ref. 2).
IRF5, a member of the IRFs family of transcription factors, is known for its activation of Toll-like receptor (TLR)-mediated innate immune responses. Expressed in B cells, dendritic cells (DCs), monocytes and macrophages (Ref. 3), IRF5 mediates antiviral response, induces IFN-dependent- and apoptosis-related gene expression and regulates the production of IFN $\alpha / \beta$ (Ref. 4). The myeloid differentiation factor 88 (MyD88)-mediated activation of IRF5 involves the formation of a tertiary complex comprising of MyD88 tetramers, TNF receptor-associated factor 6 (TRAF6), IL-1 receptor-associated kinase 1

${ }^{1}$ Department of Epidemiology and Biostatistics, School of Public Health, Anhui Medical University, 81 Meishan Road, Hefei, Anhui, 230032, PR China.

${ }^{2}$ Department of Biochemistry and Molecular Biology, University of Melbourne, VIC, Australia.

${ }^{+}$Co-senior author.

*Corresponding author: Dong-Qing Ye, Department of Epidemiology and Biostatistics, School of Public Health, Anhui Medical University, 81 Meishan Road, Hefei, Anhui, 230032, PR China. E-mail: ydq@ahmu.edu.cn 
(IRAK1) and IRF5 (Ref. 2). Recently, findings suggested that the absence of IRF5 resulted in aberrant production of auto-antibodies and proinflammatory cytokines, which correlates with the pathogenesis of SLE. Furthermore, functional analysis revealed genetic association of IRF5 and SLE. The strong association of this transcription factor with autoimmune disease on multiple levels encourages us to systematically review what had been published recently on the crucial nature of IRF5 in relation to SLE gaining attention for its regulatory capability in autoimmune lupus. The information obtained may result in a better understanding of the pathogenesis and development of novel therapeutic strategies for this systemic autoimmune disease.

\section{IRF5, a transcription factor}

Murine Irf5 gene is primarily expressed as a fulllength transcript in the $\mathrm{B}_{22} \mathrm{O}^{+}$mature $\mathrm{B}$ cells with only a single splice variant, detectable at low levels in the bone marrow (BM) (Refs 5, 6). This $\mathrm{BM} \mathrm{Mu}$ Irf5 transcript includes a 288-nucleotide deletion in the coding region, with an impaired transcriptional activity of IRF5 (Ref. 6). Both Tank binding Kinase 1 (TBK1) and MyD88 can activate the murine IRF5 to generate homodimers or activate transcription of type I IFN (Ref. 6). Furthermore, IRF5 contributes to the expression of inflammatory cytokines such as TNF $\alpha$, regulated on activation normal $\mathrm{T}$ cell expressed and secreted (RANTES) and IL-6 (Refs 6, 7, 8).

Human Irf5 gene is mapped to the chromosome $7 q 32$ (Ref. 9) (Fig. 1). There are 9 exons in Irf5 gene. It has a $\mathrm{CpG}$ island, encompassing exon $1 \mathrm{~A}$ and $1 \mathrm{~B}$ (there are four alternative $5^{\prime} \mathrm{UTR}$ exons in IRF5 gene: 1A, 1B, 1C and 1D) (Ref. 9). Of the four exons, only exon 1A transcripts are highly expressed. Therefore, it is possible that methylation of the CPG island might result in IRF5 gene being epigenetically silenced. Indeed, methylated CpG island in promoter regions has been found in lung cancer cells (increased IRF5 hyper-methylation in CRL5800, CRL5807, CRL5872 and CRL5810 cell lines) (Refs 10, 11). Allele $\mathrm{T}$ of the single nucleotide polymorphism (SNP) rs2004640 creates the $5^{\prime}$ donor splice site, causing transcription of alternative exon 1B. SNP rs10954213 is located in the polyA site AATAAA. IRF5 gene has 2 alternative polyA sites, producing transcripts with either long or short 3'UTR (Refs 12, 13, 14). mRNA with long $3^{\prime} \mathrm{UTR}$ is less stable owing to the presence of the AU-rich elements (AREs) which target mRNA for rapid degradation (Ref. 15). Exon 6 has a 30-bp in-frame indel polymorphism, which is located in a proline-, glutamic acid-, serine-, threonine-rich domain, and known to affect protein stability and function in the IRF family of proteins. Exon 6 can be spliced at an alternative site with 48-bp (SV-16) downstream of the canonical splice junction. The 30-bp insertion/ deletion (in/del-10) has distinct ability to initiate transcription of IRF5 target genes and differs from IRF5 protein isoforms in part (Refs 16, 17, 18).

\section{Correlation between IRF5 and IFNa in SLE}

IRF5 is found to be up-regulated (Ref. 19), and contributes to the heritability of serum IFN $\alpha$ activity in SLE and affects variance in IFN $\alpha$ activity (Ref. 20). Serum levels of IFN $\alpha$ were increased in SLE patients (Refs 21, 22), and associated with disease activity (Ref. 23), which makes it possible that high IFN $\alpha$ levels expressed in SLE patients might contribute to the increased IRF5 expression (Refs 17, 24). Minigene experiments demonstrated that IFN $\alpha$ could be a factor contributing to the enhanced IRF5 expression (Ref. 19). IFN $\alpha$ is known to be a pleiotropic type I IFN with the potential to break self-tolerance by activating antigen-presenting cells following uptake of self-material (Ref. 25). High levels of IFN $\alpha$ were related to the presence of anti-double-stranded DNA (anti-dsDNA) and anti-RBP antibodies (Refs 23, 26). Some patients who received recombinant human IFN $\alpha$ for the treatment of malignancy and viral hepatitis have developed de novo SLE, which typically resolves after withdrawal of the cytokine therapy (Refs 27, 28). Collectively, these findings suggest a potential role of IFN $\alpha$ in SLE susceptibility.

As noted above, high serum IFN $\alpha$ is a risk factor for SLE. Interestingly, the immune complexes (ICs) formed by auto-antibodies in SLE have been recognised to stimulate the IFN $\alpha$ synthesis by activating endosomal TLRs (Ref. 29). IRF5 downstream of endosomal TLRs may exacerbate chronic endogenous stimulation provided by ICs. Therefore, genetic variation of IRF5 may affect IFN $\alpha$ production, depending on particular autoantibodies (Ref. 30). In European ancestry patients with SLE, both anti-dsDNA and anti-Ro antibodies were associated with a risk haplotype (TACA), and anti-dsDNA correlated with a neutral haplotype (TATA), but anti-Ro antibodies were not 
Figure 1. The structure of the human interferon regulatory factor 5 (IRF5) gene. Proteins coding exons are displayed in grey and noncoding exons are shown in white. The relative positions of the single nucleotide polymorphisms (SNPs) and indel are displayed.

significantly related to the TATA haplotype, which lacked the exon 6 insertion (Table 1) (Ref. 30). Furthermore, the haplotypes related to particular auto-antibodies can lead to higher serum IFN $\alpha$ in the presence of that particular auto-antibody, suggesting that auto-antibodies are necessary for dysregulation of serum IFN $\alpha$ ascribed to IRF5 risk variants. Although in SLE patients of European American and Hispanic American ancestry, risk haplotype of IRF5 was related to the increased serum IFN $\alpha$ activity in comparison to those with protective haplotype, and this effect was prominent in patients positive for either anti-RBP or anti-dsDNA auto-antibodies (Ref. 20) (Table 1). Collectively, these findings may support a model of disease pathogenesis where specific interaction of auto-antibodies with particular IRF5 variants contributes to abnormal IFN $\alpha$ synthesis, leading to up-regulated risk of SLE (Fig. 2) (Refs 9, 30). In addition, IRF5 haplotype correlated with anti-Ro antibodies in SLE patients is associated with the same auto-antibody in healthy individuals who carry this auto-antibody, suggesting that IRF5 plays a role in serologic autoimmunity in humans (Ref. 35).
Genetic association between IRF5 and SLE Genetic association studies on SNPs in IRF5 have suggested different haplotypes that confer either susceptibility to, or protection from SLE (Refs 31, 36, 37). Three putative functional alleles (rs2004640, rs10954213 and exon 6 indel) were believed to be associated with SLE in European descent (Ref. 31). When combined, these alleles and another SNP, rs2070197, haplotype 1 was significantly associated with the risk of SLE, and the risk haplotype 1 was predicted to express exon 1B isoform (because of rs2004640) (Ref. 31) (Table 1). Haplotypes 4 and 5 indicated strong evidence for protection. Moreover, individuals who carried haplotype 1 in trans with either of the haplotypes (haplotyes 4 or 5) which lacked the expression of exon 1B isoform displayed a down-regulation of SLE susceptibility. Thus, the highest risk to SLE for a haplotype is predicted to be expressed at high level transcripts containing exon 1B. As for the haplotypes in SLE patients in Sweden, the risk haplotype contains the risk alleles of the four polymorphisms: 4xCGGGG indel Ins, rs2004640 T, rs10954213 A and rs10488631 C, whereas the 
Table 1. Association of IRF5 haplotypes with SLE

\begin{tabular}{|c|c|c|c|}
\hline Reference & SNP & Haplotype & Population \\
\hline 19 & $\begin{array}{l}\text { rs2004640( }\left(T^{a} / G^{b}\right) \\
\text { rs10954213( }\left(A^{a} / G^{b}\right) \\
\text { rs10488631(C } \\
\text { indel }\left(\text { Ins }^{a} / \mathrm{Del}^{\mathrm{b}}\right)\end{array}$ & $\begin{array}{l}\text { Risk haplotype: rs2004640 } \\
\text { (T)-rs10954213 (A)- } \\
\text { rs10488631 (C)-CGGGG indel } \\
\text { (Ins) } \\
\text { Protective haplotype: } \\
\text { rs2004640 (G)-rs10954213 } \\
\text { (G)-rs10488631 (T)-CGGGG } \\
\text { indel (Del) }\end{array}$ & Sweden \\
\hline 20 & $\begin{array}{l}\text { rs2004640( }\left(T^{\mathrm{a}} / \mathrm{G}^{\mathrm{b}}\right), \mathrm{rs} 3807306\left(\mathrm{~A}^{\mathrm{a}} /\right. \\
\left.\mathrm{C}^{\mathrm{b}}\right), \mathrm{rs} 2280714\left(\mathrm{~A}^{\mathrm{a}} / \mathrm{G}^{\mathrm{b}}\right), \\
\left.\text { rs10488631( } \mathrm{C}^{\mathrm{a}} / \mathrm{T}^{\mathrm{b}}\right)\end{array}$ & $\begin{array}{l}\text { Risk haplotype: rs2004640 } \\
\text { (T)-rs3807306 (A)-rs10488631 } \\
\text { (C)-rs2280714 (A) } \\
\text { Protective haplotype: } \\
\text { rs2004640 (G)-rs3807306 (C)- } \\
\text { rs10488631 (T)-rs2280714 (A) }\end{array}$ & $\begin{array}{l}\text { European } \\
\text { American, } \\
\text { Hispanic American }\end{array}$ \\
\hline 30 & $\begin{array}{l}\text { rs2004640 }\left(\mathrm{T}^{\mathrm{a}} / \mathrm{G}^{\mathrm{b}}\right), \mathrm{rs} 3807306\left(\mathrm{~A}^{\mathrm{a}} /\right. \\
\left.\mathrm{C}^{\mathrm{b}}\right), \mathrm{rs} 2280714\left(\mathrm{~A}^{\mathrm{a}} / \mathrm{G}^{\mathrm{b}}\right), \\
\left.\text { rs10488631( } \mathrm{C}^{\mathrm{a}} / \mathrm{T}^{\mathrm{b}}\right)\end{array}$ & $\begin{array}{l}\text { Risk haplotype (TACA): } \\
\text { rs2004640 (T)-rs3807306 (A)- } \\
\text { rs10488631 (C)-rs2280714 (A) } \\
\text { Neutral haplotype (TATA): } \\
\text { rs2004640 (T)-rs3807306 (A)- } \\
\text { rs10488631 (T)-rs2280714 (A) }\end{array}$ & European \\
\hline 31 & $\begin{array}{l}\text { rs2004640 }\left(T^{a} / G^{b}\right) \text {, exon } 6 \\
\text { indel }\left(\operatorname{Ins}^{a} / D^{b} l^{b}\right), \text { rs2070197( } C^{a} / \\
\left.T^{b}\right), \text { rs10954213( }\left(A^{a} / G^{b}\right)\end{array}$ & $\begin{array}{l}\text { Haplotype } 1 \text { (risk): rs2004640 } \\
\text { (T)-exon } 6 \text { indel (Ins)- } \\
\text { rs2070197 (C)-rs10954213 (A) } \\
\text { Haplotype } 4 \text { (protective): } \\
\text { rs2004640 (G)-exon } 6 \text { indel } \\
\text { (Ins)-rs2070197 (T)- } \\
\text { rs10954213 (G) } \\
\text { Haplotye } 5 \text { (protective): } \\
\text { rs2004640 (G)-exon } 6 \text { indel } \\
\text { (Del)-rs2070197 (T)- } \\
\text { rs10954213 (A) }\end{array}$ & European \\
\hline 32 & $\begin{array}{l}\text { rs2004640( }\left(T^{a} / G^{b}\right) \\
\text { rs } 10954213\left(A^{a} / G^{b}\right) \\
\text { rs752637( } \\
\left.C^{b}\right), \text { rs2280714 }\left(A^{\mathrm{a}} / G^{b}\right)\end{array}$ & $\begin{array}{l}\text { Risk haplotype: rs2004640 } \\
\text { (T)-rs752637 (G)-rs3807306 } \\
\text { (A)-rs10954213 (A)- } \\
\text { rs2280714 (A) }\end{array}$ & African American \\
\hline 33 & 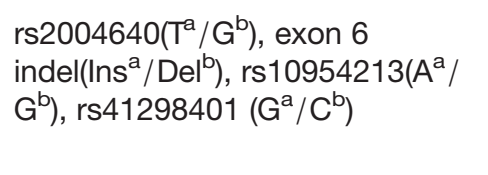 & $\begin{array}{l}\text { Protective haplotype: } \\
\text { rs2004640 (G)-rs41298401 } \\
\text { (G)-rs10954213 (A)-exon } 6 \\
\text { indel (Del) }\end{array}$ & Japanese \\
\hline 34 & $\begin{array}{l}\text { rs2004640 }\left(T^{a} / G^{b}\right), \\
\text { rs10954213(A }\left(A^{a} / G^{b}\right)\end{array}$ & $\begin{array}{l}\text { Risk haplotype: rs2004640 } \\
\text { (T)-rs10954213 (A) } \\
\text { Protective haplotype: } \\
\text { rs2004640 (G)-rs10954213 } \\
\text { (G) }\end{array}$ & $\begin{array}{l}\text { European } \\
\text { American }\end{array}$ \\
\hline \multicolumn{4}{|c|}{$\begin{array}{l}\text { aRisk allele. } \\
\text { bProtective allele. } \\
\text { Del, deletion; Ins, insertion; IRF5, interferon regulatory factor 5; SNP, single nucleotide polymorphism; SLE, } \\
\text { systemic lupus erythematosus. }\end{array}$} \\
\hline
\end{tabular}




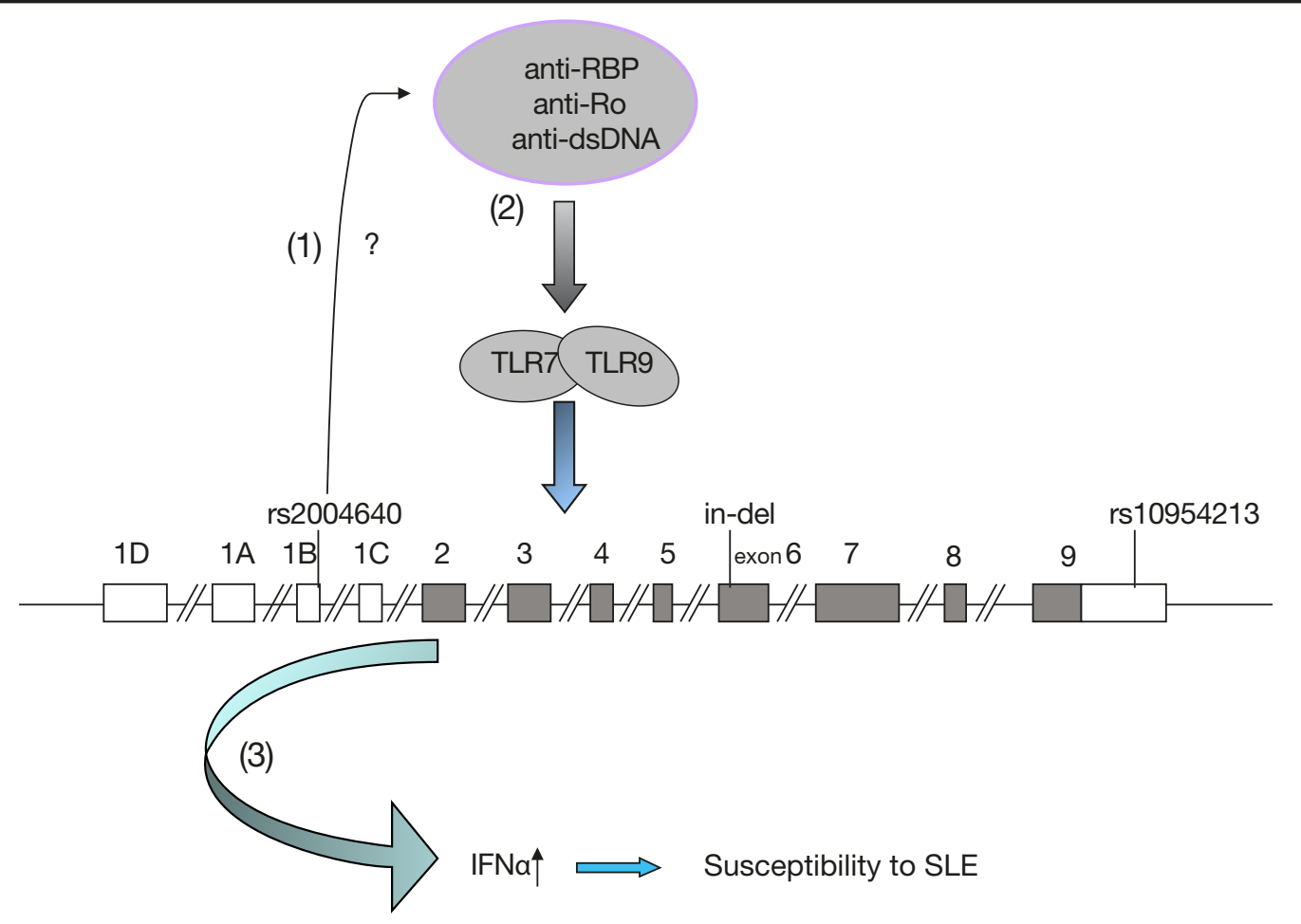

Relationships among the proposed association of IRF5 haplotypes, IFNa and SLE-specific auto-antibodies in the pathogenesis of SLE.

Expert Reviews in Molecular Medicine (c) 2013 Cambridge University Press

Figure 2. Relationships among the proposed association of interferon regulatory factor 5 (IRF5) haplotypes, IFNa and Systemic lupus erythematosus (SLE)-specific auto-antibodies in the pathogenesis of SLE. (1) ?: IRF5 haplotypes may affect autoimmune susceptibility to generate particular auto-antibodies. (2) These auto-antibodies (anti-RBP, anti-Ro and anti-dsDNA) in cells may trigger endosomal Toll-like receptor $7 / 9$ (TLR7/9). (3) Specific IRF5 related risk variants enhance downstream IFNa synthesis in the context of auto-antibodies, leading to high serum IFNa and SLE susceptibility.

corresponding protective haplotype contains the nonrisk alleles of the polymorphisms (Table 1) (Ref. 19). With respect to the haplotype structure of IRF5 in African Americans, results of five variants (rs2004640, rs752637, rs3807306, rs10954213 and rs2280714) revealed a significant haplotype (TGAAA) determined by the minor A risk allele at rs3807306 (Table 1) (Ref. 32). Multivariate analysis demonstrated that rs3807306 was required for the association with SLE because all other variants, individually, and the haplotype, as a unit lost significance, when conditioned on rs3807306. In another study on SLE in Japanese population, a protective haplotype carrying rs2004640 G, rs41298401 G, a deletion in exon 6 and rs10954213 A, defined by the intron 1 SNP rs41298401, was identified (Ref. 33) (Table 1). Collectively, these findings suggest that IRF5 haplotypes were significantly associated with SLE in multiple ethnicities.

TLRs are able to recognise endogenous nucleic acids in the context of ICs detected in SLE patients, which promotes DCs maturation and IFN $\alpha$ synthesis, a process that involves IRF5 (Ref. 38). A TATA-less promoter region, designated P1, was mapped in Epstein-Barr virus (EBV) BamHI-A rightward transcripts, where P1 had relatively high basal activity in B cells. After EBV infection of B cells, transcription from P1 was detected soon upon infection, but IRF5 negatively regulated P1 activity (Ref. 39). In addition, transient expression of IRF5 decreased activation of the EBV oncoprotein 1 (LMP1) promoter, and transfection of either an IRF5 dominant-negative construct or IRF5 small interfering RNA in type II EBV cells (C1D12 and 
C2G6) led to up-regulation in endogenous levels of LMP1, suggesting that IRF5 can down-regulate the expression of LMP1 (Ref. 40). Furthermore, EBV infection of type III latently infected B-cell lines LCL, Mutu III and EREB2-5 induced IRF5 expression, and examination of IRF-5 transcripts revealed a novel splice variant, V12, which was induced by EBV infection, constitutively localised in nuclear, performed as a dominant negative form in IRF-5 (Ref. 41). These data indicate that EBV is significantly related to IRF5. EBV infection is known to be associated with SLE susceptibility (Refs 42, 43, 44, 45). Since B cells are the primary host cell type for EBV infection, it is likely that IRF5 affects B cell responses (B cell receptor crosslinking) to cause the disease. Compared with the protective haplotype (Table 1), peripheral blood mononuclear cells (PBMCs) in SLE patients from European American descents with the risk haplotype (both stimulated and un-stimulated with EBV) indicated three pathways with significance: the INF, TLR and B cell receptor (BCR) pathways (Ref. 34). IFN-induced transmembrane protein 1 (IFITM1) had higher expression in cells with risk haplotype than cells without risk haplotype at steady states (Ref. 34). Fos and MyD88 were both under-expressed in the un-stimulated cells with risk haplotype compared with the cells with protective haplotype (Ref. 34). In addition, CD79A and CD79B were decreased 2.2-fold in the uninfected cells with risk haplotype, but this difference disappeared after EBV infection. Collectively, these data suggest that IRF5 haplotype may be a key determinant in not only the IFN, TLR pathways, but also the BCR pathways in SLE.

Recently, the A allele of rs10954213 was found to be related to the high level of IRF5 in SLE patients (Ref. 14). In mice, mutations disrupting AREs in the tumor necrosis factor $\alpha(\mathrm{TNF} \alpha)$ gene can result in up-regulation of circulating TNF $\alpha$ and hypersensitivity in the presence of stimulation with TLR4 ligand lipopolysaccharide (LPS) (Refs 46, 47). Therefore, it is possible that rs10954213 in the 3'UTR polyA site of IRF5 might be a functional mutation required for high levels of IRF5. IRF5 expression and alternative splicing were significantly increased in SLE patients compared with healthy controls, especially when the transcription from exon 1A and exon 1D was markedly up-regulated in the patient cells (Ref. 19). It is known that increased serum levels of small nuclear ribonucleoproteins (snRNPs) and serine/arginine-rich (SR) proteins, which are components of the spliceosome, can be found in SLE patients (Refs 48, 49). Following co-transfection with snRNPs or SR proteins, the transcription from exon $1 \mathrm{~A}$ and alternative splicing of exon 5-7 were enhanced. In particular, co-transfection with U1 snRNP led to the strongest increase of IRF5 expression, implying its contribution to the enhanced alternative splicing (Ref. 19). Furthermore, IRF5 transcript expression was higher in patients carrying the risk haplotype versus patients carrying the protective haplotype, and IRF5 protein expression was increased in myeloid DCs and monocytes from SLE patients, correlating with the risk haplotype (Ref. 19). In addition, the functional effects of exon 6 in/del10 and SV-16 within IRF5 were demonstrated in SLE, where the expression of either SV-16 or in/ del-10 confers IRF5 the ability to impair apoptotic response, and is related to the downregulation of IRF5 nuclear translocation in murine embryonic fibroblasts (MEFs) after treatment with DNA-damaging stimulus (Ref. 50). MEFs that expressed IRF5-bearing SV16 displayed up-regulated IL-6 production upon LPS stimulation. By contrast, MEFs containing in/del-10 produced IL-6 with no significant difference from the controls. Intriguingly, the absence or presence of both in/del-10 and SV-16 leads to abrogation of these effects. Together, these observations indicate that specific genetic architecture of IRF5 correlates with the upregulated expression of IRF5 in SLE patients, and IRF5 variants carried on SLE risk haplotypes modulate IRF5 function.

\section{IRF5 and the immune mechanisms of autoimmune lupus}

It has been observed that compared with the nonlupus-prone strain of mice (C57BL/6), lupusprone mice [NZB, $\left.(\mathrm{NZB} / \mathrm{W}) \mathrm{F}_{1}, \quad \mathrm{NZM} 2410\right]$ produced higher levels of IRF5 mRNA in their splenic cells (Ref. 5). In nonautoimmune mice, IRF5 deficiency reduced the expression of Blimp1, IFN $\beta$ and p202 proteins, whereas the levels of Aim 2 and $F c \gamma$ RIIB proteins were up-regulated. On the contrary, overexpression of IRF5 in RAW264.7 cells can rescue these defective effects whereas the levels of IRF5 mRNA and proteins were increased in Aim2 $2^{-/-}$and Fc $\gamma \mathrm{RIIB}^{-/-}$mice (Ref. 51). In lupus-susceptible B6.Nba2 and 
B6.Sle123 congenic mice, the expression of IRF5 mRNA and proteins as well as IFN $\beta$ mRNA from their splenic cells were up-regulated compared with the age-matched B6 mice, which was positively related to the increased expression of Blimp-1 and p202 proteins, but negatively associated with the levels of Aim2 and Fc $\gamma$ RIIB proteins (Ref. 51). These data demonstrated that IRF5 regulates the expression of lupus susceptibility genes, and implied that IRF5 may contribute to lupus susceptibility in part by down-regulating the expression of aim2 and $f_{c} \gamma$ riib genes (Refs 51, 52, 53). Since the aberrant expression of IRF5 in human and murine cells is associated with SLE as discussed above, and IRF5 exhibits pathogenic potential by controlling immune response, advanced insight of regulatory mechanism of IRF5 in immune cells becomes critical to understand the pathogenesis and develops better therapeutic strategies for SLE (Ref. 54).

\section{The role of IRF5 in B cells}

IRF5 plays an important role in B cell function. It promotes $B$ cell maturation in part by modulating the expression of Blimp-1, a master regulator of plasma cell maturation (Ref. 55). IRF5 deficiency in mice resulted in an altered isotype switching, leading to diminished IgG2a/c responses to TI and TD antigens and to virus infection (Ref. 4). In responding to immunisation with NP-KLH, a TD antigen, IgG1 response was markedly lower in IRF5 ${ }^{-1-}$ mice compared with the control mice (Ref. 4). On the contrary, the virus-specific antibody response of IRF5 ${ }^{-1-}$ mice to the major polyoma virus (PyV) capsomer protein VP1 demonstrated small difference in the levels of antiviral IgG1 in comparison with the control mice. Similarly, stimulation of $\mathrm{IRF}^{-/-}$B cells with LPS and IL-4 led to upgraded levels of IgG1 germline transcripts as well as IgG1-expressing B cells (Ref. 4). Adoptive transfer experiments indicated that the attenuated $\operatorname{IgG} 2 \mathrm{a} / \mathrm{c}$ response is an intrinsic property of IRF5 $5^{-1-} \mathrm{B}$ cells.

It is well-known that class switch recombination (CSR) can be induced in B cells in vitro by combination of multiple B cell activators, such as TLR ligands and cytokines (Ref. 56). Purified $B$ cells from naive IRF5 ${ }^{-1-}$ mice induced less preferential switching to $\operatorname{IgG} 2 \mathrm{a} / \mathrm{c}$ than that of wild-type mice cultured with LPS alone or cocultured with IL-4, or IFN $\gamma$ (Ref. 2). These findings were demonstrated by the fact that modulation of the $\operatorname{IgG} 2 \mathrm{a} / \mathrm{c}$ responses was mediated by the IRF5-Ikaros (ikzf1) axis. Evidence has indicated that IRF5 mediates induction of $\operatorname{IgG2a}$ in virtue of controlling Ikaros expression (Ref. 57). The Ikaros family of transcription factors is able to silence their target genes through recruitment of transcriptional inhibitor complexes, for instance, histone deacetylase complexes. Ikzf1 ${ }^{-1-}$ mice cannot generate $\mathrm{B}$ cells (Ref. 58), whereas $\mathrm{Ikzf1}^{\mathrm{L} / \mathrm{L}}$ mice ('L' for Lac Z) up-regulated their production of IgG2a/c during LPS- and IFN $\gamma$-induced CSR in culture (Refs 59, 60). The levels of ikzf1 transcripts were elevated in $\mathrm{IRF}^{-1-} \mathrm{B}$ cells undergoing CSR, indicating that the expression of ikzf1 was regulated by IRF5, which was confirmed by sequence analysis where there was an IRF-binding site in the promoter of the $i k z f 1$ gene that specifically bound IRF5 in the presence of MyD88 (Ref. 4). Together, these results identify the IRF5-Ikaros axis as a critical modulator of IgG2a/c class switching in B cells (Fig. 3).

IRF5 deficiency in pristane-induced lupus mice did not produce IgG2b, IgG2c, IgG3, IgM antiU1A Abs, anti-Sm/RNP auto-antibodies, antinuclear antibodies (ANA) and anti-dsDNA auto-antibodies (Refs 3, 61, 62). Similarly, the production of auto-antibodies, including ANA, anti-dsDNA antibody, anti-Sm antibody and anti-RNP antibody, was dramatically attenuated in MRL/lpr, Fc $\gamma$ RIIB $^{-/-}$Yaa and Fc $\gamma$ RIIB $^{-/-}$ lupus mice deficient in IRF5 (Refs 61, 62, 63, 64). When the correlation between the absence of auto-antibodies and defective isotype switching was evaluated in IRF5 ${ }^{-/-}$mice, the pristanetreated IRF5 ${ }^{-1-}$ mice produced decreased levels of IgG2b, IgG2c and IgG1 anti-U1A Abs (Ref. 61). However, IgM levels were comparable between IRF5 ${ }^{-1-}$ and control mice (Ref. 61). These results were later confirmed by the findings that IRF5 ${ }^{-1-}$ mice revealed significant decreases in both IgG2a/c and IgG2b levels (Refs 3, 62). Therefore, the down-regulation of $\operatorname{IgG} 2 \mathrm{a} / \mathrm{c}$ and $\operatorname{IgG} 2 \mathrm{~b}$ may be associated with significant decreases in specific lupus autoantibodies. In the pristane-induced lupus mice, IRF5 deficiency indicated upgraded serum levels of total IgG1, implying that IRF5 deficiency does not decrease long-lived IgG1 expressing plasma cells (Ref. 62). After class switching, autoreactive $B$ cells might undergo further selection and expansion, where IRF5 ${ }^{-/-}$mice were lack 


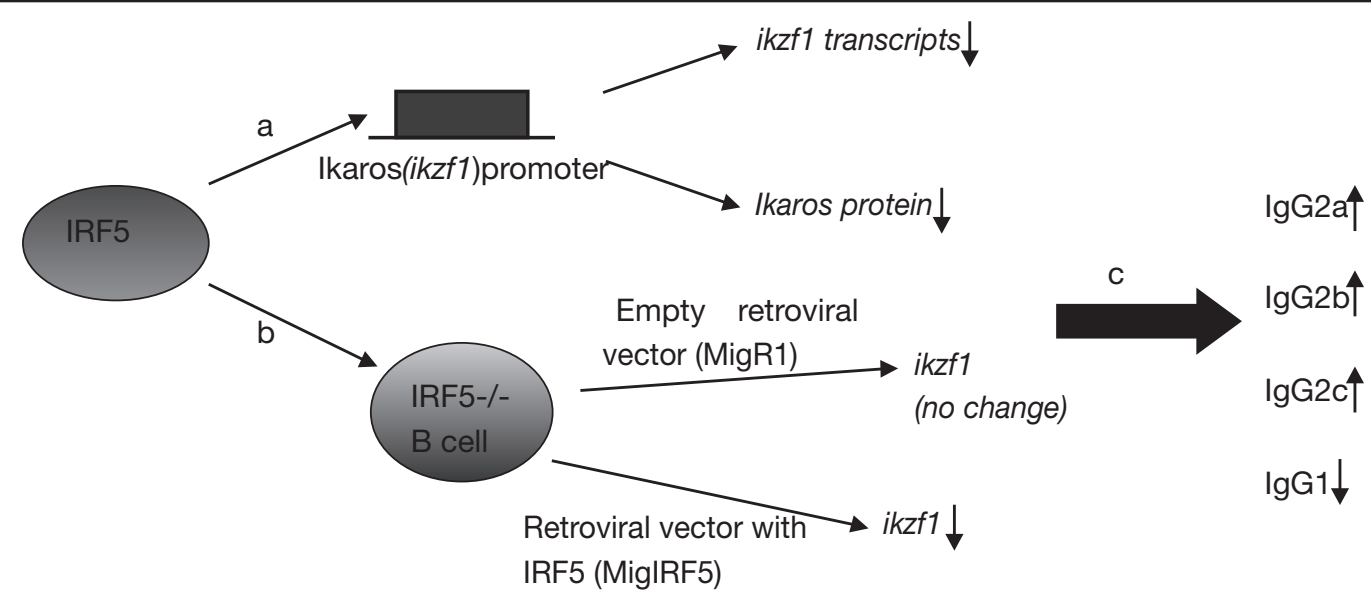

Relationships of IRF5-Ikaros-Isotype class switch B cells.

Expert Reviews in Molecular Medicine ( 2013 Cambridge University Press

Figure 3. Relationships of interferon regulatory factor 5 (IRF5)-Ikaros-Isotype class switch B cells. (a) IRF5 binds to ikzf1 promoter, results in Ikaros (ikzf1) transcripts and proteins down-regulation. (b) Transduction of IRF5 deficient B cells with empty retroviral vector (MigR1), or IRF5 (MigIRF5), leads to no changed ikzf1 or decreased ikzf1 transcripts, respectively. (c) IRF5 negatively regulates Ikaros (ikzf1), which may up-regulate the levels of $\lg G 2 a, \lg G 2 b$ and $\operatorname{lgG} 2 c$, but down-regulate the levels of lgG1.

of the generation of IgG1 auto-antibodies, implying that a mechanism other than class switching controls antigen specificity in these mice. Instead, IRF5 might be of importance for selection or expansion of auto-reactive clones from B cell repertoire (Refs 61, 62). However, the clear mechanism is not known to date, which needs further investigation.

When the role of type I IFN in inducing IgG2a secretion by IRF5 $5^{+/+}$and IRF5 ${ }^{-/-}$B cells was investigated, it was found that exogenous type I IFN did not rescue the defect in CpG-B (a TLR9 ligand)-induced IgG2a secretion in the absence of IRF5, in spite of their response to type I IFN (Ref. 3). Furthermore, there were no differences in the levels of type I IFN mRNA between CpGB-stimulated IRF5 ${ }^{+/+}$and IRF5 ${ }^{-/-}$B cells. Thus, these results suggest that IRF5 may regulate IgG2a synthesis via its ability to control type I IFN gene expression, which was verified by the absence of IgG2a production in type I IFN receptor-deficient B cells stimulated with CpG-B (Ref. 3). Moreover, IRF5 was found to be necessary for IL-6 production in B cells (Ref. 8). Indeed, IL-6 is critical for the generation of antidsDNA auto-antibodies in pristane-injected lupus mice (Ref. 65). Furthermore, the expression of ccl3 and ccl4 mRNA were compromised in
TLR9-stimulated IRF5 ${ }^{-1-}$ B cells, suggesting a role of IRF5 in orchestrating inflammatory responses (Ref. 3). In addition, IRF5 ${ }^{-1-}$ B cells proliferated less in response to TLR9 stimulation than IRF5 $^{+/+}$B cells did. Collectively, this evidence suggested that IRF5 may regulate B cell activation following TLR9 stimulation.

\section{IRF5 in T (Th) cells}

Fc $\gamma$ RIIB deficient mice can induce a spontaneous SLE-like disease, this property of the $\mathrm{Fc} \gamma \mathrm{RIIB}^{-/-}$ B6 model mimics the multigenic nature of human SLE (Refs 63, 66, 67). Addition of the Yaa locus to this model leads to a significant increase in severity of the disease (Ref. 67). In the Yaa murine lupus model, IRF5 was critical for T cell activation, because the expression of the activation markers CD69 and CD44 on splenic T cells was significantly reduced in $\mathrm{IRF5}^{-/-}$ RII.Yaa mice compared with IRF5 ${ }^{+/+}$RII.Yaa mice (Ref. 63). In pristane-induced lupus mice, the expression of CD69 on splenic CD4 ${ }^{+} \mathrm{T}$ cells of IRF5 ${ }^{-1-}$ mice was markedly down-regulated (Ref. 62), whereas in MRL/lpr mice, analysis of spleen cell populations revealed that the memory (CD62 $\left.\mathrm{L}^{\text {low }} \mathrm{CD} 44^{\text {high }}\right)$ and activated $\left(\mathrm{CD} 62 \mathrm{~L}^{\text {high }} \mathrm{CD} 44^{\text {high }}\right) \mathrm{CD}^{+} \mathrm{T}$ cells were decreased in comparison to $\mathrm{B}_{2} 20^{+}$activated 
$\mathrm{CD}^{+}{ }^{+}$T cells (Ref. 57). Therefore, IRF5 is important in $\mathrm{T}$ cell activation in lupus mice.

Consistent with the importance of type I IFN in the pathogenesis of lupus, IRF5 ${ }^{-1-}$ mice produced significantly reduced levels of the ISGs IRF7, ISG15 and Mx1 (Refs 61, 62). Meanwhile, protein levels of the type I IFN-inducible chemokine MCP-1 (CCL2), pro-inflammatory cytokines such as IL-12 p40/70, IL-6 and IL23p19 were also down-regulated in $\mathrm{IRF}^{-/-}$ mice (Ref. 61). These data suggest that the deficiency of IRF5 attenuated lupus by influencing the production of type I IFN and other cytokines that combine innate immunity with the activation of T-helper 1 (Th1) and Th17 cells (Ref. 68). On the contrary, serum levels of Th2 cytokines IL-4, IL-5 and IL-10 were increased in $\mathrm{IRF}^{-/-}$mice compared with $\mathrm{IRF5}^{+/+}$mice (Ref. 62). The upgraded Th2 cytokine may contribute to the disease protection in $\mathrm{IRF}^{-/-}$mice since Th2 cells promote the synthesis of the least pathogenic IgG1 isotype observed in IRF5 ${ }^{-1-}$ mice (Ref. 69). To interpret the $\mathrm{T}$ cell identity that generated Th2 cytokines from pristane-induced IRF5 ${ }^{-/-}$ mice, the authors found that $\mathrm{CD} 4^{+} \mathrm{T}$ cells from IRF5 $^{+/+}$mice generated negligible amounts of IL-4 and only Th1 cells can be detected in $\mathrm{IRF}^{+/+}$mice (Ref. 62). In the IRF5 ${ }^{-/-}$mice, however, a readily noticeable fraction of IL-4 was produced. These findings support the fact that IRF5 is critical in the regulation of Th1/Th2 polarisation, and contributes to pristane-induced lupus pathogenesis. $\mathrm{Xu}$ et al (Ref. 61) investigated Th cell subsets in type I IFN receptor (IFNAR) deficient and TLR7 deficient mice and confirmed that IL-4-producing cells had decreased. Furthermore, in IRF5 ${ }^{-/-}$mice, the ratio of Th1 to Th2 was lower in IFNAR deficient and TLR7 deficient mice than their wild-type counterparts. On the other hand, serum levels of IL-6 were reduced in IRF5 ${ }^{-1-}$ RII.Yaa mice, but the levels of IFN $\gamma$ were comparable between IRF5 ${ }^{-1-}$ RII.Yaa mice and controls (Ref. 63). Hence, IRF5 deficiency cannot completely attenuate all the autoimmune phenotypes.

All type I IFNs act via a single cell surface type I IFN receptor, termed IFNAR (IFNAR1 and IFNAR2) (Refs 70, 71, 72, 73). Both spleen and lymph node sizes were smaller in IFNAR1 ${ }^{-/-}$ RII.Yaa mice relative to IFNAR $1^{+/+}$RII.Yaa mice (Ref. 63). Renal diseases were less severe in the IFNAR $1^{-/-}$RII.Yaa mice compared with the
IFNAR $1^{+/+}$RII.Yaa mice as well, which were demonstrated by reduction in glomerular crescent formation, cell number per glomerulus and interstitial disease (Ref. 63). Furthermore, IFNAR1 $1^{-/-}$RII.Yaa mice survived longer than IFNAR $1^{+/+}$RII.Yaa mice. These findings may suggest a role of type I IFN in driving the pathogenesis of SLE. Expression of type I IFNregulated genes IFIT1 and MX2 was similar in ${\mathrm{B} 220^{+}}$splenocytes from IRF5 ${ }^{+/+}$or $\mathrm{IRF5}^{-/-}$ RII.Yaa mice in comparison with wild-type mice (Ref. 63). By contrast, kidneys from IRF5 ${ }^{+/+}$ RII.Yaa mice showed 3-fold induction of both IFIT1 and MX2, suggesting the evidence for IRF5-dependent type I IFN expression, albeit at low levels and only at a site of severe inflammation. It is notable that serum levels of IL-6 and IL-10 were decreased in IRF5 ${ }^{-/-}$ RII.Yaa mice (Ref. 63), and it has been known that down-regulation of IL-6 and IL-10 might be relevant for lupus pathogenesis, where these two cytokines can contribute to B cell activation and auto-antibody synthesis, and are related to disease activity in lupus patients (Ref. 73). Therefore, it is likely that IRF5 effects are medicated through IFNAR independent pathways in this lupus model, possibly through suppression of the generation of IL- 6 and IL-10.

Influence of IRF5 on DC immune responses IRF5 plays an important role in DC (Fig. 4). IRF5 ${ }^{-1-}$ and $\mathrm{MyD} 88^{-1-}$ mice treated with pristane had strikingly reduced number of $\mathrm{CD}_{11 \mathrm{~b}}{ }^{-} \mathrm{CD} 11 \mathrm{C}^{+} \mathrm{B} 220^{+} \mathrm{PDCA}^{+}{ }^{+}$pDCs in the peritoneal cavity compared with wild-type $\mathrm{B} 6$ mice (Ref. 61). However, the percentage of pDCs in the BM was similar between IRF5 ${ }^{-1-}$ and wild-type mice. CCR7 is expressed on DCs, and its ligands (CCL19 and CCL21) are required for homing of pDCs to lymph nodes (Ref. 74). The expression of both CCL19 and CCL21 in peritoneal exudates cells from IRF5 ${ }^{-1-}$ mice were lower than that of control mice (Ref. 61). In light of the MyD88 dependent pDC homing and the ability of TLR ligands to promote CCR7 expression (Ref. 74), fluorescence intensity of

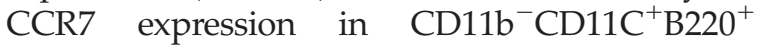
PDCA $-1^{+}$pDCs was found to be comparable in $\mathrm{BM}$ between $\mathrm{B} 6$ and $\mathrm{IRF} 5^{-/-}$mice, and the $\mathrm{IRF5}^{-/-}$peritoneal $\mathrm{pDCs}$ displayed similar fluorescence intensity to that of $\mathrm{IRF}^{-/-} \mathrm{BM}$ pDCs (Ref. 61). Consequently, these data may support the fact that MyD88 expression promotes 


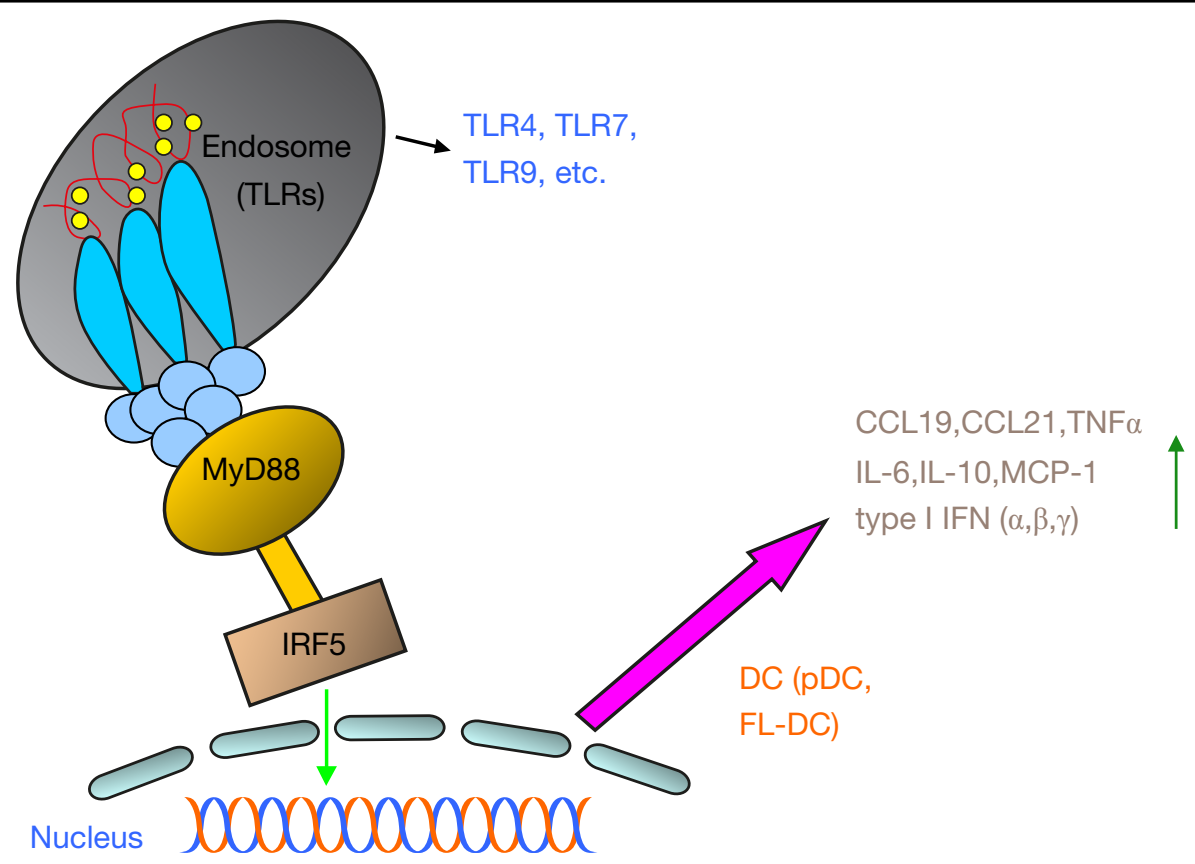

\section{Possible relationships between IRF5-chemokines and cytokines in} dendritic cells (DCs).

Expert Reviews in Molecular Medicine (C) 2013 Cambridge University Press

Figure 4. Possible relationships between interferon regulatory factor 5 (IRF5)-chemokines and cytokines in dendritic cells (DCs). Endosomal TLRs (such as TLR4, TLR7, TLR9, etc.) recruit MyD88, subsequently bind to and stimulate IRF5, leading to activated pro-inflammatory cytokine and chemokine production in DCs (pDC, FL-DC).

pDC migration to the peritoneum by up-regulating IRF5-regulated CCL19/CCL21 but not CCR7 expression.

In splenic DCs from MRL/lpr mice, IRF5 deficiency generated reduced levels of $\mathrm{TNF} \alpha$, IL-6 and IL-10 in response to the TLR9 ligand CpG and TLR7 ligand loxorbine (Ref. 64). The levels of IFN $\alpha$, IFN $\gamma$ and MCP-1 were declined in response to $\mathrm{CpG}$ as well. Since ICs were able to activate DCs through engagement of TLRs and Fcy receptors (Refs 75, 76, 77, 78), stimulation of wild-type DCs with purified ICs from the serum of MRL/lpr mice produced upgraded levels of TNF $\alpha$ and IL-6, whereas ICsinduced cytokine production was markedly decreased in the DCs from $\mathrm{IRF}^{-/-}$mice (Ref. 64). These findings indicate that IRF5 mediates the activation of DCs that are triggered by ICs or by TLR7 or TLR9 engagement, and IRF5 deficiency may cause a deficit in proinflammatory cytokines generation. Moreover, LPS-induced IFN $\alpha$ synthesis in IFN $\beta$-pretreated FL-DCs (DCs obtained from BM cells cultured in fms-like tyrosine kinase 3 ligand) was found to be dependent on TLR4 with sequential involvement of the TRIF (Toll/IL-1 receptor domain-containing adaptor protein-inducing IFN $\beta$ ) and MyD88 pathways (Ref. 79). In the FLDCs from IRF5 ${ }^{-/-}$mice, LPS-induced IFN $\alpha$ synthesis was not completely down-regulated as compared with wild-type FL-DCs. However, both CpG-A- and TLR3 ligand poly(I:C)induced IFN $\alpha$ production were severely downregulated in $\mathrm{IRF} 5^{-/-}$mice, implying a critical role of IRF5 in IFN $\alpha$ production via MyD88and TRIF-dependent pathways. 
Since IFN $\beta$ and IFN $\alpha$ share the same receptor and activate similar IFN $\alpha \beta$-inducible gene programs (Ref. 80 ), it is likely that IFN $\beta$ may potentially contribute to the effects of type I IFN on lupus that were generally ascribed to that of IFN $\alpha$. Lupus IgG, conventional TLR7 ligand R848 and CPG-A stimulation induced appreciable IFN $\beta$ production by FL-DCs, which is dependent on IRF5 (Ref. 38). Moreover, compared with wild-type control, activation of FL-DCs from IRF5 ${ }^{-1-}$ mice by lupus IgG and CpG-A (50 nM) induced substantially reduced production of IFN $\alpha$ (Ref. 38). This is in contrast to what other people have observed that IFN $\alpha$ synthesis in pDC induced by CpG-A at $1 \mu \mathrm{M}$ was IRF5 independent (Ref. 65). This discrepancy may be attributable to several reasons: for example, the issue of dosage might be related to lupus pathogenesis where the activation strength of TLR7 or TLR9 by nucleic acid-containing ICs is weaker than that elicited by maximal doses of microbial and synthetic loxorbine, CpG-A. Besides, it is also likely that pDCs from spleen in vivo are different from the pDCs in FL-DCs culture in their requirement for IRF5 in CPG-A induced IFN $\alpha$ synthesis. Recently, IRF5 ${ }^{-/-}$mice have been found with impaired pDC development, which is not dependent on IRF5 (Ref. 81). IFN $\alpha$ secretion by IRF5 $^{-1-}$ pDCs following CpG-A stimulation was similar to background levels (Ref. 67). A genomic duplication within Dock2 led to a frame-shift mutation and premature stop codon, and the ectopic expression of DOCK2 by retroviral transduction restored $\mathrm{pDCs}$ development. Still, IRF5 ${ }^{-1-}$ mice lacking the Dock2 mutation had largely normal IFN $\alpha$ synthesis from pDCs (Ref. 81). Therefore, a reduction in the expression of Dock2 may be responsible for the impaired pDCs in $\mathrm{IRF5}^{-1-}$ mice. Interestingly, Yasuda et al. (Ref. 82) found that IRF5 is involved in the TLR9-induced type I IFN production independent of the Dock2 mutation, showing with reduced IFN $\alpha$ and IFN $\beta$ production in IRF5 ${ }^{-/-}$FL-DCs upon stimulation with TLR9 ligands. However, IRF5 ${ }^{-1-}$ mice with the Dock2 mutation showed higher serum levels of IgG1 and lower levels of IgG2b, IgG2a/c and IgG3 than IRF5 ${ }^{-1-}$ mice without the Dock2 mutation, suggesting that the Dock2 mutation contributes to Th2-type effects (Ref. 82). These findings need to be clarified in lupus in the future.

\section{Conclusion}

The understanding of the immune-pathological mechanism of SLE is gradually evolving with budding studies on evaluating the important role of IRF5 in controlling T, B lymphocytes and DCs, as well as the underlying intracellular signalling mechanisms in each of these immune cell types in response to various stimuli. A number of methodologies including gene expression, familial cytokine analysis and genetic association studies were employed to demonstrate the over-active IFN $\alpha$ pathway as a critical factor in the pathogenesis of SLE. IRF5 is a crucial mediator of IFN $\alpha$ and IFN $\alpha$-induced gene expression, and correlates with endosomal TLRs activation (Ref. 61). These TLRs are pathogenically activated by SLE-related, autoimmune complexes, which must have provided a chronic endogenous stimulus to the IFN $\alpha$ pathway in SLE. Detailed studies supporting IRF5 gene polymorphisms are gainof-function, leading to some of the IFN $\alpha$ pathway activation observed in the disease. Nevertheless, whether the observed gene/autoantibody interaction upon IFN $\alpha$ stimulation is dependent on other parts of the SLE phenotype is not clear to date (Ref. 83). Furthermore, EBV infection is known to involve SLE pathogenesis, and viral infection may supply a strong stimulus to the TLRs/IRF5 system. Therefore, a model of 'gene + microenvironment stimulus = high IFN $\alpha$ ' may still apply. However, with the advent of more advanced technology and studies, our understanding of the role of IRF5 in this elusive disease is expected to be further enriched with novel findings.

\section{Acknowledgement}

This work was partly supported by grants from the key program of National Natural Science Foundation of China (30830089), National Natural Science Foundation of China (81102192, 81172764) and National Health and Medical Research Council Project Grant of Australia (1006428).

\section{Conflict of interest}

The authors declare no conflict of interest.

\section{References}

1 Xu, W.D. et al. (2012) IRF7, a functional factor associates with systemic lupus erythematosus. Cytokine 58, 317-320 
2 Tamura, T. et al. (2008) The IRF family transcription factors in immunity and oncogenesis. Annual Review of Immunology 26, 535-584

3 Savitsky, D.A. et al. (2010) Contribution of IRF5 in B cells to the development of murine SLE-like disease through its transcriptional control of the IgG2a locus. Proceedings of the National Academy of Sciences of the United States of America 107, 10154-10159

4 Fang, C.M. et al. (2012) Unique contribution of IRF-5Ikaros axis to the B-cell IgG2a response. Genes and Immunity 13, 421-430

5 Shen, H. et al. (2010) Gender-dependent expression of murine Irf5 gene: implications for sex bias in autoimmunity. Journal of Molecular Cell Biology 2, 284-290

6 Paun, A. et al. (2008) Functional characterization of murine interferon regulatory factor 5 (IRF-5) and its role in the innate antiviral response. Journal of Biological Chemistry 283, 14295-14308

7 Yanai, H. et al. (2007) Role of IFN regulatory factor 5 transcription factor in antiviral immunity and tumor suppression. Proceedings of the National Academy of Sciences of the United States of America 104, 3402-3407

8 Takaoka, A. et al. (2005) Integral role of IRF-5 in the gene induction programme activated by Toll-like receptors. Nature 434, 243-249

9 Kozyrev, S.V. and Alarcon-Riquelme, M.E. (2007) The genetics and biology of Irf5-mediated signaling in lupus. Autoimmunity 40, 591-601

$10 \mathrm{Li}$, Q. and Tainsky, M.A. (2011) Epigenetic silencing of IRF7 and/or IRF5 in lung cancer cells leads to increased sensitivity to oncolytic viruses. PLoS ONE 6, e28683

11 Kondo, Y. and Issa, J.P. (2010) DNA methylation profiling in cancer. Expert Reviews in Molecular Medicine 12, e23

12 Kyogoku, C. et al. (2006) Functional characterization of the systemic lupus erythematosus risk gene IRF-5. In American College of Rheumatology Annual Meeting, http://www.rheumatologyorg/

13 Cunninghame et al. (2007) Association of IRF5 in UK SLE families identifies a variant involved in polyadenylation. Human Molecular Genetics 16, 579-591

14 Kozyrev, S.V. et al. (2007) Structural insertion/ deletion variation in IRF5 is associated with a risk haplotype and defines the precise IRF5 isoforms expressed in systemic lupus erythematosus.

Arthritis Rheumatism 56, 1234-1241

15 Khabar, K.S. (2005) The AU-rich transcriptome: more than interferons and cytokines, and its role in disease. Journal of Interferon and Cytokine Research 25, 1-10
16 Barnes, B.J. et al. (2004) Global and distinct targets of IRF-5 and IRF-7 during innate response to viral infection. Journal of Biological Chemistry 279, 45194-45207

17 Mancl, M.E. et al. (2005) Two discrete promoters regulate the alternatively spliced human interferon regulatory factor-5 isoforms. Multiple isoforms with distinct cell type-specific expression, localization, regulation, and function. Journal of Biological Chemistry 280, 21078-21090

18 Barnes, B.J. et al. (2002) Multiple regulatory domains of IRF-5 control activation, cellular localization, and induction of chemokines that mediate recruitment of T lymphocytes. Molecular and Cellular Biology 22, 5721-5740

19 Feng, D. et al. (2010) Genetic variants and diseaseassociated factors contribute to enhanced IRF-5 expression in blood cells of systemic lupus erythematosus patients. Arthritis Rheumatism 62, 562-573

20 Niewold, T.B. et al. (2008) Association of the IRF5 risk haplotype with high serum interferon-alpha activity in systemic lupus erythematosus patients. Arthritis Rheumatism 58, 2481-2487

21 Hooks, J.J. et al. (1979) Immune interferon in the circulation of patients with autoimmune disease. New England Journal of Medicine 301, 5-8

22 Baechler, E.C. et al. (2003) Interferon-inducible gene expression signature in peripheral blood cells of patients with severe lupus. Proceedings of the National Academy of Sciences of the United States of America 100, 2610-2615

23 Kirou, K.A. et al. (2005) Activation of the interferonalpha pathway identifies a subgroup of systemic lupus erythematosus patients with distinct serologic features and active disease. Arthritis Rheumatism 52, 1491-1503

24 Barnes, B.J. et al. (2003) IRF-5, a novel mediator of cell-cycle arrest and cell death. Cancer Research 63, 6424-6431

25 Blanco, P. et al. (2001) Induction of dendritic cell differentiation by IFN- $\alpha$ in systemic lupus erythematosus. Science 294, 1540-1543

26 Weckerle, C.E. et al. (2011) Network analysis of associations between serum interferon- $\alpha$ activity, autoantibodies, and clinical features in systemic lupus erythematosus. Arthritis Rheumatism 63, 1044-1053

27 Rönnblom, L.E., Alm, G.V. and Oberg, K.E. (1990) Possible induction of systemic lupus erythematosus by interferon-alpha treatment in a patient with a malignant carcinoid tumour. Journal of Internal Medicine 227, 207-210 
28 Niewold, T.B. and Swedler, W.I. (2005) Systemic lupus erythematosus arising during interferonalpha therapy for cryoglobulinemic vasculitis associated with hepatitis C. Clinical Rheumatology 24, 178-181

29 Lövgren, T. et al. (2004) Induction of interferon-alpha production in plasmacytoid dendritic cells by immune complexes containing nucleic acid released by necrotic or late apoptotic cells and lupus IgG. Arthritis Rheumatism 50, 1861-1872

30 Niewold, T.B. et al. (2012) IRF5 haplotypes demonstrate diverse serological associations which predict serum interferon alpha activity and explain the majority of the genetic association with systemic lupus erythematosus. Annals of the Rheumatic Diseases 71, 463-468

31 Graham, R.R. et al. (2007) Three functional variants of IFN regulatory factor 5 (IRF5) define risk and protective haplotypes for human lupus.

Proceedings of the National Academy of Sciences of the United States of America 104, 6758-6763

32 Kelly, J.A. et al. (2008) Interferon regulatory factor-5 is genetically associated with systemic lupus erythematosus in African Americans. Genes and Immunity 9, 187-194

33 Kawasaki, A. et al. (2008) Association of IRF5 polymorphisms with systemic lupus erythematosus in a Japanese population: support for a crucial role of intron 1 polymorphisms. Arthritis Rheumatism 58, 826-834

34 Guthridge, J.M. et al. (2012) Effects of IRF5 lupus risk haplotype on pathways predicted to influence B cell functions. Journal of Biomedicine and Biotechnology 2012, 594056

35 Cherian, T.S. et al. (2012) Brief Report: IRF5 systemic lupus erythematosus risk haplotype is associated with asymptomatic serologic autoimmunity and progression to clinical autoimmunity in mothers of children with neonatal lupus. Arthritis Rheumatism 64, 3383-3387

36 Sigurdsson, S. et al. (2005) Polymorphisms in the tyrosine kinase 2 and interferon regulatory factor 5 genes are associated with systemic lupus erythematosus. American Journal of Human Genetics 76, 528-537

37 Graham, R.R. et al. (2006) A common haplotype of interferon regulatory factor 5 (IRF5) regulates splicing and expression and is associated with increased risk of systemic lupus erythematosus. Nature Genetics 38, 550-555

38 Yasuda, K. et al. (2007) Murine dendritic cell type I IFN production induced by human IgG-RNA immune complexes is IFN regulatory factor (IRF)5 and IRF7 dependent and is required for IL-6 production. Journal of Immunology 178, 6876-6885

39 Chen, H. et al. (2005) Regulation of expression of the Epstein-Barr virus BamHI-A rightward transcripts. Journal of Virology 79, 1724-1733

40 Ning, S., Huye, L.E. and Pagano, J.S. (2005) Interferon regulatory factor 5 represses expression of the Epstein-Barr virus oncoprotein LMP1: braking of the IRF7/LMP1 regulatory circuit. Journal of Virology 79, 11671-11676

41 Martin, H.J. et al. (2007) Manipulation of the toll-like receptor 7 signaling pathway by Epstein-Barr virus. Journal of Virology 81, 9748-9758

42 James, J.A., Harley, J.B. and Scofield, R.H. (2006) Epstein-Barr virus and systemic lupus erythematosus. Current Opinion in Rheumatology 18, 462-467

43 James, J.A. et al. (1997) An increased prevalence of Epstein-Barr virus infection in young patients suggests a possible etiology for systemic lupus erythematosus. Journal of Clinical Investigation 100, 3019-3026

44 James, J.A. et al. (2001) Systemic lupus erythematosus in adults is associated with previous Epstein-Barr virus exposure. Arthritis Rheumatism $44,1122-1126$

45 Lu, J.J. et al. (2007) Association of Epstein-Barr virus infection with systemic lupus erythematosus in Taiwan. Lupus 16, 168-175

46 Jacob, C.O., Lee, S.K. and Strassmann, G. (1996) Mutational analysis of TNF- $\alpha$ gene reveals a regulatory role for the $3^{\prime}$-untranslated region in the genetic predisposition to lupus-like autoimmune disease. Journal of Immunology 156, 3043-3050

47 Kontoyiannis, D. et al. (1999) Impaired on/off regulation of TNF biosynthesis in mice lacking TNF AU-rich elements: implications for joint and gutassociated immunopathologies. Immunity 10, 387-398

48 Lerner, M.K. and Steitz, J.A. (1979) Antibodies to small nuclear RNAs complexed with proteins are produced by patients with systemic lupus erythematosus. Proceedings of the National Academy of Sciences of the United States of America 76, 5495-5499

49 Neugebauer, K.M. et al. (2000) SR proteins are autoantigens in patients with systemic lupus erythematosus. Arthritis Rheumatism 43, 1768-1778

50 Wen, F. et al. (2011) Exon 6 variants carried on systemic lupus erythematosus (SLE) risk haplotypes modulate IRF5 function. Autoimmunity 44, 82-89

51 Panchanathan, R. et al. (2012) Distinct regulation of murine lupus susceptibility genes by the IRF5/ Blimp-1 axis. Journal of Immunology 188, 270-278 
52 Panchanathan, R. et al. (2010) Aim2 deficiency stimulates the expression of IFN-inducible Ifi202, a lupus susceptibility murine gene within the $\mathrm{Nba} 2$ autoimmune susceptibility locus. Journal of Immunology 185, 7385-7393

53 Panchanathan, R. et al. (2011) Cell type and genderdependent differential regulation of the p202 and Aim2 proteins: implications for the regulation of innate immune responses in SLE. Molecular Immunology 49, 273-280

$54 \mathrm{Xu}$, W.D. et al. (2012) Targeting IRF4 in autoimmune diseases. Autoimmunity Reviews 11, 918-924

55 Lien, C. et al. (2010) Critical role of IRF-5 in regulation of B-cell differentiation. Proceedings of the National Academy of Sciences of the United States of America 107, 4664-4668

56 Stavnezer, J. (2000) Molecular processes that regulate class switching. Current Topics in Microbiology and Immunology 245, 127-168

57 Kim, J. et al. (1999) Ikaros DNA-binding proteins direct formation of chromatin remodeling complexes in lymphocytes. Immunity 10, 345-355

58 Wang, J.H. K. et al. (1996) Selective defects in the development of the fetal and adult lymphoid system in mice with an Ikaros null mutation. Immunity 5, 537-549

59 Kirstetter, P. et al. (2002) Ikaros is critical for B cell differentiation and function. European Journal of Immunology 32, 720-730

60 Sellars, M. et al. (2009) Ikaros controls isotype selection during immunoglobulin class switch recombination. Journal of Experimental Medicine 206, 1073-1087

$61 \mathrm{Xu}$, Y. et al. (2012) Pleiotropic IFN-dependent and -independent effects of IRF5 on the pathogenesis of experimental lupus. Journal of Immunology 188, 4113-4121

62 Feng, D. et al. (2012) Irf5-deficient mice a re protected from pristane-induced lupus via increased Th2 cytokines and altered IgG class switching. European Journal of Immunology 42, 1477-1487

63 Richez, C. et al. (2010) IFN regulatory factor 5 is required for disease development in the FcgammaRIIB - /- Yaa and FcgammaRIIB -/mouse models of systemic lupus erythematosus. Journal of Immunology 184, 796-806

64 Tada, Y. et al. (2011) Interferon regulatory factor 5 is critical for the development of lupus in MRL/lpr mice. Arthritis Rheumatism 63, 738-748

65 Richards, H.B. et al. (1998) Interleukin 6 dependence of anti-DNA antibody production: evidence for two pathways of autoantibody formation in pristaneinduced lupus. Journal of Experimental Medicine $188,985-990$
66 Bolland, S. and Ravetch, J.V. (2000) Spontaneous autoimmune disease in $\mathrm{Fc}$ (gamma)RIIB-deficient mice results from strain-specific epistasis. Immunity 13, 277-285

67 Bolland, S. et al. (2002) Genetic modifiers of systemic lupus erythematosus in FcgammaRIIB(-/-) mice. Journal of Experimental Medicine 195, 1167-1174

68 Hunter, C.A. (2005) New IL-12-family members: IL-23 and IL-27, cytokines with divergent functions. Nature Reviews of Immunology 5, 521-531

69 Takahashi, S. et al. (1996) Imbalance towards Th1 predominance is associated with acceleration of lupus-like autoimmune syndrome in MRL mice. Journal of Clinical Investigation 97, 1597-1604

70 Oritani, K. et al. (2001) Type I interferons and limitin: a comparison of structures, receptors, and functions. Cytokine Growth Factor Reviews 12, 337-348

71 Fenner, J.E. et al. (2006) Suppressor of cytokine signaling 1 regulates the immune response to infection by a unique inhibition of type I interferon activity. Nature Immunology 7, 33-39

72 Uzé, G. et al. (2007) The receptor of the type I interferon family. Current Topics in Microbiology Immunology 316, 71-95

73 Illei, G.G. et al. (2004) Biomarkers in systemic lupus erythematosus: II. Markers of disease activity. Arthritis Rheumatism 50, 2048-2065

74 Seth, S. et al. (2011) CCR7 essentially contributes to the homing of plasmacytoid dendritic cells to lymph nodes under steady-state as well as inflammatory conditions. Journal of Immunology 186, 3364-3372

75 Boulé, M.W. et al. (2004) Toll-like receptor 9-dependent and -independent dendritic cell activation by chromatin-immunoglobulin $\mathrm{G}$ complexes. Journal of Experimental Medicine 199, $1631-1640$

76 Means, T.K. et al. (2005) Human lupus autoantibodyDNA complexes activate DCs through cooperation of CD32 and TLR9. Journal of Clinical Investigation $115,407-417$

77 Savarese, E. et al. (2006) U1 small nuclear ribonucleroprotein immune complexes induce type I interferon in plasmacytoid dendritic cells through TLR7. Blood 107, 3229-3234

78 Leadbetter, E.A. et al. (2002) Chromatin-IgG complexes activate B cells by dual engagement of IgM and Toll-like receptors. Nature 416, 603-607

79 Richez, C. et al. (2009) TLR4 ligands induce IFNalpha production by mouse conventional dendritic cells and human monocytes after IFN-beta priming. Journal of Immunology 182, 820-828 
80 Theofilopoulos, A.N. et al. (2005) Type I interferons $(\alpha / \beta)$ in immunity and autoimmunity. Annual Review of Immunology 23, 307-336

81 Purtha, W.E. et al. (2012) Spontaneous mutation of the Dock2 gene in Irf5 - /- mice complicates interpretation of type Iinterferon production and antibody responses. Proceedings of the National Academy of Sciences of the United States of America 109, E898-E904
82 Yasuda, K. et al. (2013) Phenotype and function of $B$ cells and dendritic cells from interferon regulatory factor 5-deficient mice with and without a mutation in DOCK2. International Immunology 25, 295-306

83 Salloum, R. and Niewold, T.B. (2011) Interferon regulatory factors in human lupus pathogenesis. Translation Research 157, 326-331

\section{Further reading}

Niewold, T.B. et al. (2008) Association of the IRF5 risk haplotype with high serum interferon-alpha activity in systemic lupus erythematosus patients. Arthritis Rheumatism 58, 2481-2487.

This article shows the biologic relevance of the SLE risk haplotype of IRF5 at the protein level.

Niewold, T.B. et al. (2012) IRF5 haplotypes demonstrate diverse serological associations which predict serum interferon alpha activity and explain the majority of the genetic association with systemic lupus erythematosus. Annals of the Rheumatic Diseases 71, 463-468.

This study indicates the association between IRF5 haplotypes, IFNa and SLE-specific auto-antibodies in the pathogenesis of SLE.

Lien, C. et al. (2010) Critical role of IRF-5 in regulation of B-cell differentiation. Proceedings of the National Academy of Sciences of the United States of America 107, 4664-4668.

This study demonstrates that IRF-5 is involved in B-cell maturation and the stimulation of Blimp-1 expression.

Savitsky, D.A. et al. (2010) Contribution of IRF5 in B cells to the development of murine SLE-like disease through its transcriptional control of the lgG2a locus. Proceedings of the National Academy of Sciences of the United States of America 107, 10154-10159.

This article demonstrates a requirement for IRF5 in development of murine SLE via its role in B lymphocytes.

\begin{tabular}{l}
$\qquad$ Features associated with this article \\
\hline Figures \\
Figure 1. The structure of the human interferon regulatory factor 5 (IRF5) gene. \\
Figure 2. Relationships among the proposed association of interferon regulatory factor 5 (IRF5) haplotypes, \\
IFNa and Systemic lupus erythematosus (SLE)-specific auto-antibodies in the pathogenesis of SLE. \\
Figure 3. Relationships of interferon regulatory factor 5 (IRF5)-lkaros-Isotype class switch B cells. \\
Figure 4. Possible relationships between interferon regulatory factor 5 (IRF5)-chemokines and cytokines in \\
dendritic cells (DCs). \\
Table \\
Table 1. Association of IRF5 haplotypes with SLE.
\end{tabular}

\section{Citation details for this article}

Wang-Dong Xu, Hai-Feng Pan, Yuekang Xu and Dong-Qing Ye (2013) Interferon regulatory factor 5 and autoimmune lupus. Expert Rev. Mol. Med. Vol. 15, e6, July 2013, doi:10.1017/erm.2013.7 


\section{University Library}

\section{- M M I E R R A A gateway to Melbourne's research publications}

Minerva Access is the Institutional Repository of The University of Melbourne

Author/s:

Xu, W-D;Pan, H-F;Xu, Y;Ye, D-Q

Title:

Interferon regulatory factor 5 and autoimmune lupus

Date:

2013-07-24

Citation:

Xu, W. -D., Pan, H. -F., Xu, Y. \& Ye, D. -Q. (2013). Interferon regulatory factor 5 and autoimmune lupus. EXPERT REVIEWS IN MOLECULAR MEDICINE, 15, https:// doi.org/10.1017/erm.2013.7.

Publication Status:

Accepted manuscript

Persistent Link:

http://hdl.handle.net/11343/41825 